\title{
Antisense yycG modulates the susceptibility of Staphylococcus aureus to hydrogen peroxide via the sarA
}

\author{
Shizhou Wu' ${ }^{1}$ Yunjie Liu², Lei Lei ${ }^{3^{*}}$ and Hui Zhang ${ }^{*^{*}}$
}

\begin{abstract}
Background: The infectious pathogen Staphylococcus aureus (S. aureus) is primarily associated with osteomyelitis. Hydrogen peroxide drainage is an effective antimicrobial treatment that has been adopted to combat $S$. aureus infections. Previous investigations have indicated that the antisense RNA (asRNA) strategy negatively modulates $S$. aureus YycFG TCS, and it significantly disrupts biofilm formation. However, the effects of the antisense yycG RNA (ASyycG) strategy on the susceptibility of biofilm-producing $S$. aureus to hydrogen peroxide and the mechanisms underlying this effect have not been elucidated to date.

Results: Overexpression of ASyycG inhibited the transcription of biofilm formation-related genes, including sarA and icaA. Additionally, the CFU counts and the live bacterial ratios of ASyycG biofilm-producing S. aureus treated with $\mathrm{H}_{2} \mathrm{O}_{2}$ were notably reduced across the groups. Notably, the predicted promoter regions of the sarA and icaA genes were directly regulated by YycF.

Conclusions: ASyycG was observed to sensitize biofilm-producing S. aureus to $\mathrm{H}_{2} \mathrm{O}_{2}$ intervention synergistically via the sarA and thus may represent a supplementary strategy for managing osteomyelitis. However, future in-depth studies should attempt to replicate our findings in animal models, such as the rat osteomyelitis model.
\end{abstract}

Keywords: Antisense, YycFG, Hydrogen peroxide, Staphylococcus aureus, sarA

\section{Background}

In humans, Staphylococcus aureus (S. aureus) is associated with a variety of diseases, ranging from relatively mild skin and soft tissue infections to life-threatening bacteremia and endocarditis. Hydrogen peroxide drainage is an effective antimicrobial treatment to combat $S$. aureus colonization and invasion [1]. Notably, it is well-known that 1000 -fold greater resistance to antimicrobial agents was observed in the biofilm state than in the planktonic

\footnotetext{
*Correspondence: leilei@scu.edu.cn; caesarzh@163.com

${ }^{3}$ Department of Preventive Dentistry, Hospital of Stomatology, State Key Laboratory of Oral Diseases, Sichuan University, NO.14 Third Section, Renmin South Road, Sichuan 610041 Chengdu, P.R. China

'Department of Orthopedics, West China Hospital, Sichuan University, No.37 Guoxue Alley, Sichuan 610041 Chengdu, P.R. China

Full list of author information is available at the end of the article
}

state [2-5]. During the establishment of biofilms, polysaccharide intercellular adhesion (PIA) is considered to be the key mechanism that provides an essential scaffold for biofilms and a special kind of extracellular matrix that is encoded by the ica operon and sarA [6, 7]. The sarA gene has been reported to drive biofilm formation by altering ica transcription and producing PIA [2, 4]. In Staphylococcus epidermidis (S. epidermidis), SarA was reported to be an essential positive regulator of the ica operon in biofilm development [8]. Additionally, numerous studies have evaluated the regulatory pathway of the sarA gene and demonstrated that $\operatorname{sar} A$ was associated with bacterial oxidation sensing, virulence factors, and biofilm formation in S. aureus [9-11].

Two-component signal transduction systems (TCSs) are essential regulators of staphylococcal metabolism

(C) The Author(s). 2021 Open Access This article is licensed under a Creative Commons Attribution 4.0 International License, which permits use, sharing, adaptation, distribution and reproduction in any medium or format, as long as you give appropriate credit to the original author(s) and the source, provide a link to the Creative Commons licence, and indicate if changes were made. The images or other third party material in this article are included in the article's Creative Commons licence, unless indicated otherwise in a credit line to the material. If material is not included in the article's Creative Commons licence and your intended use is not permitted by statutory regulation or exceeds the permitted use, you will need to obtain permission directly from the copyright holder. To view a copy of this licence, visit http://creativecommons.org/licenses/by/4.0/ The Creative Commons Public Domain Dedication waiver (http://creativecommons.org/publicdomain/zero/1.0/) applies to the data made available in this article, unless otherwise stated in a credit line to the data. 
and adaptation to environmental changes [12, 13]. Among the 16 known TCSs in S. aureus, YycFG is essential for bacterial viability [14]. The YycG histidine kinase is generally anchored by a cytoplasmic membrane and is involved in monitoring environmental changes. Notably, when the responses of this kinase to extracellular stimuli transfer the phosphoryl group to activate the response regulator (RR) YycF, the cellular physiology status, including biofilm organization, adapts to changes $[7,14,15]$. In a previous study, we demonstrated that the essential YycFG TCS was closely related to biofilm formation and extracellular matrix organization [16]. However, since the YycFG TCS is essential to S. aureus viability, the construction of deletion mutants of YycFG was unsuccessful [17].

Antisense RNA (asRNA) is a single-stranded RNA that is complementary to the target messenger RNA (mRNA). The interaction between the RNA molecules inhibits downstream signal transduction activation. Using this asRNA strategy, we constructed mutant antisense $S$. aureus strains that express YycFG TCS at low levels. The number of icaA gene transcripts and the level of biofilm formation decreased in the ASyycG-mutant $S$. aureus, and YycFG was expressed at low levels [18]. However, the potential mechanisms by which the transcriptional regulator $\mathrm{YycF}$ modulates sarA and/or icaA expression and the biological effects of this modulation on the susceptibility of biofilm-producing $S$. aureus to hydrogen peroxide warrant further research.

In the present study, we investigated the role played by antisense $y y c G$ in regulating the susceptibility of biofilm-producing $S$. aureus to $\mathrm{H}_{2} \mathrm{O}_{2}$ treatment. In addition, the potential association of $\mathrm{YycF}$ with sarA and icaA was evaluated using an electrophoretic mobility shift assay (EMSA). This work primarily investigated whether an antisense $y y c G$ interference strategy could sensitize biofilm-producing $S$. aureus to $\mathrm{H}_{2} \mathrm{O}_{2}$ intervention, which may be associated with the direct regulation of YycF to the adjacent genes sarA and icaA.

\section{Results}

\section{Antisense yycG modulated oxidative regulation}

Transcriptome and enrichment analyses showed that overexpression of antisense $y y c G$ influenced the pathways associated with biofilm metabolism, virulence, oxidative regulation, and glycolysis/gluconeogenesis utilization by S. aureus (Fig. 1a and b). Notably by padj values ranking, the 20 KEGG pathways with the greatest differential expression that were involved in S. aureus infection and metabolism were determined to have enrichment score mostly at around 0.5 differences between the antisense $y y c G$ overexpression group and ATCC29213 group. The less padj value was, the greater expression diversity in pathway between the antisense $y y c G$ overexpression group and ATCC29213 group. Each pathway involved different genes count, such as microbial metabolism in diverse environments involving about 60 genes with a padj value at about 0.05 (Fig. 1b). Moreover, three genes that regulate biofilm formation and virulence were modulated; specifically, sarA was downregulated, and $\operatorname{cod} Y$ and $\operatorname{srr} A$ were upregulated (see Fig. 1c). These results indicated that antisense $y y c G$ negatively regulated biofilm formation and the expression of the virulence-associated gene $\operatorname{sar} A$.

\section{Antisense yycG sensitized biofilm-producing $S$. aureus to $\mathrm{H}_{2} \mathrm{O}_{2}$ intervention synergistically}

Through fluorescence microscopy, the live ratios of bacteria were compared. In the ASyycG $+\mathrm{H}_{2} \mathrm{O}_{2}$ group (Fig. 2a), the antibacterial effect of $\mathrm{H}_{2} \mathrm{O}_{2}$ was significantly enhanced by ASyyc $G$ synergistically and exhibited the lowest live bacteria ratio at $22.1 \pm 4.6$ compared with each group (Fig. $2 b$ ). The SEM and immunofluorescence results were similar and exhibited the lowest EPS levels in the ASyyc $+\mathrm{H}_{2} \mathrm{O}_{2}$ group. In addition, we could only detect rare cell clusters in ASyycG $+\mathrm{H}_{2} \mathrm{O}_{2}$, even after $24 \mathrm{~h}$ of culture (Fig. 3a). Quantitively, we performed a CFU test on each biofilm. Correspondingly, the CFU count of AS S. aureus $+\mathrm{H}_{2} \mathrm{O}_{2}$ biofilm was mostly lower in all groups than in the $S$. aureus ATCC29213 parent group (Fig. 3b). Accordingly, we evaluated the ability of the S. aureus strains to form biofilms. The biomass was quantified via a microtiter dish assay, and ASyycG strains exhibited reduced biofilm formation compared with the S. aureus ATCC29213 group. In particular, the ASyycG strains treated with $\mathrm{H}_{2} \mathrm{O}_{2}$ exhibited the strongest decrease in biofilm formation among all groups, which indicated that AS S. aureus $+\mathrm{H}_{2} \mathrm{O}_{2}$ biofilms had significantly decreased biofilm growth compared to the other groups (Fig. 4a). These results showed that antisense $y y c G$ could significantly enhance the susceptibility of $S$. aureus to $\mathrm{H}_{2} \mathrm{O}_{2}$ and exert negative effects on biofilm-producing $S$. aureus after $\mathrm{H}_{2} \mathrm{O}_{2}$ treatment.

\section{Antisense $y y c G$ overexpression inhibited the transcription of the sarA and ica genes}

Quantitative RT-PCR analyses demonstrated that the expression levels of the genes in the YycFG pathway, including $y y c G$ and $y y c F$, were significantly reduced, which may be attributable to the overexpression of $A S y y c G$ in the ASyycG group treated with $\mathrm{H}_{2} \mathrm{O}_{2}$. Furthermore, the expression levels of sarA related to the oxide stress reaction and biofilm-associated ica genes were more significantly reduced by $\mathrm{ASyycG}$ overexpression in the ASyycG strains treated with $\mathrm{H}_{2} \mathrm{O}_{2}$ than in the $S$. aureus parent ATCC29213 strains $(\mathrm{n}=10, P<0.05$; Fig. 4b). In keeping with this result, YycFG pathway protein expression was significantly downregulated in the ASyycG group treated or not treated with $\mathrm{H}_{2} \mathrm{O}_{2}$ compared with the 
A

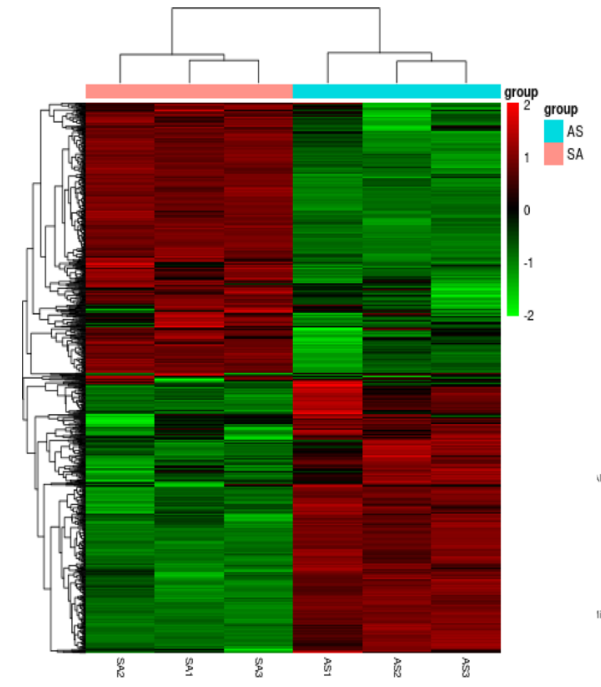

B

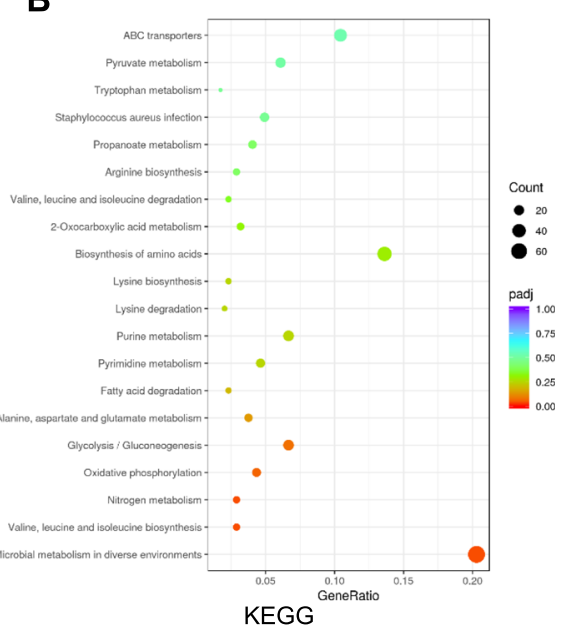

C

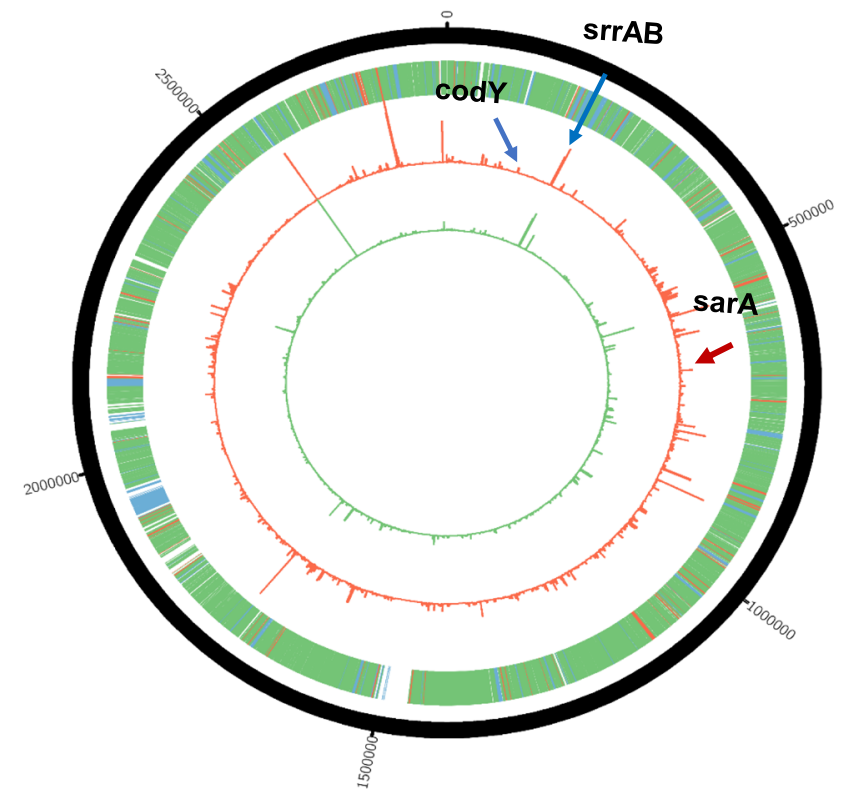

Fig. 1 Transcriptome analysis of the antisense yycG modulation. a Heatmap for the Transcriptome analysis; b KEGG annotation statistics; $\mathbf{c}$ Genomic map showing transcription profiles of ATCC29213 and antisense yycG mutant strains. The middle (red) circle depicts FPKM values for antisense yycG mutant cells, and the inner (green) circle depicts FPKM values for the ATCC29213 strains. The outer (yellow) circle is a heat map showing fold changes in expression between the ATCC29213 and the antisense yycG mutant

ATCC29213 strain. Notably, the protein expression levels of the cognate sensor kinase $\mathrm{YycG}$ and the response regulator $\mathrm{YycF}$ were elevated in response to $\mathrm{H}_{2} \mathrm{O}_{2}$ intervention (Fig. 4c). These results indicated that antisense $y y c G$ inhibited the expression of the YycFG pathway, which may have contributed to reductions in downstream icaA and sarA gene expression.

\section{YycF bound to the predicted promoter regions of sarA and icaA genes}

The purified recombinant $\mathrm{YycF}$ protein was visualized via Coomassie staining after SDS-PAGE (Fig. 4d). The promoter region of sarA contained a putative YycF binding consensus motif (Fig. 5a). We employed the bacterial promoter database (http://www.softberry.com/berry. phtml? topic $=$ index \& group $=$ programs $\&$ subgroup $=$ gfindb), and a putative promoter of the sarA gene was predicted. EMSA demonstrated that the YycF protein was bound to DNA fragments from sarA and icaA promoter regions. As a negative control, to rule out nonspecific binding, we utilized a DNA fragment of the same size as the predicted promoter and with a similar AT: $\mathrm{GC}$ mole ratio but missing the $\mathrm{YycF}$ consensus binding sequence (Fig. 5b). By EMSAs, we determined that YycF 


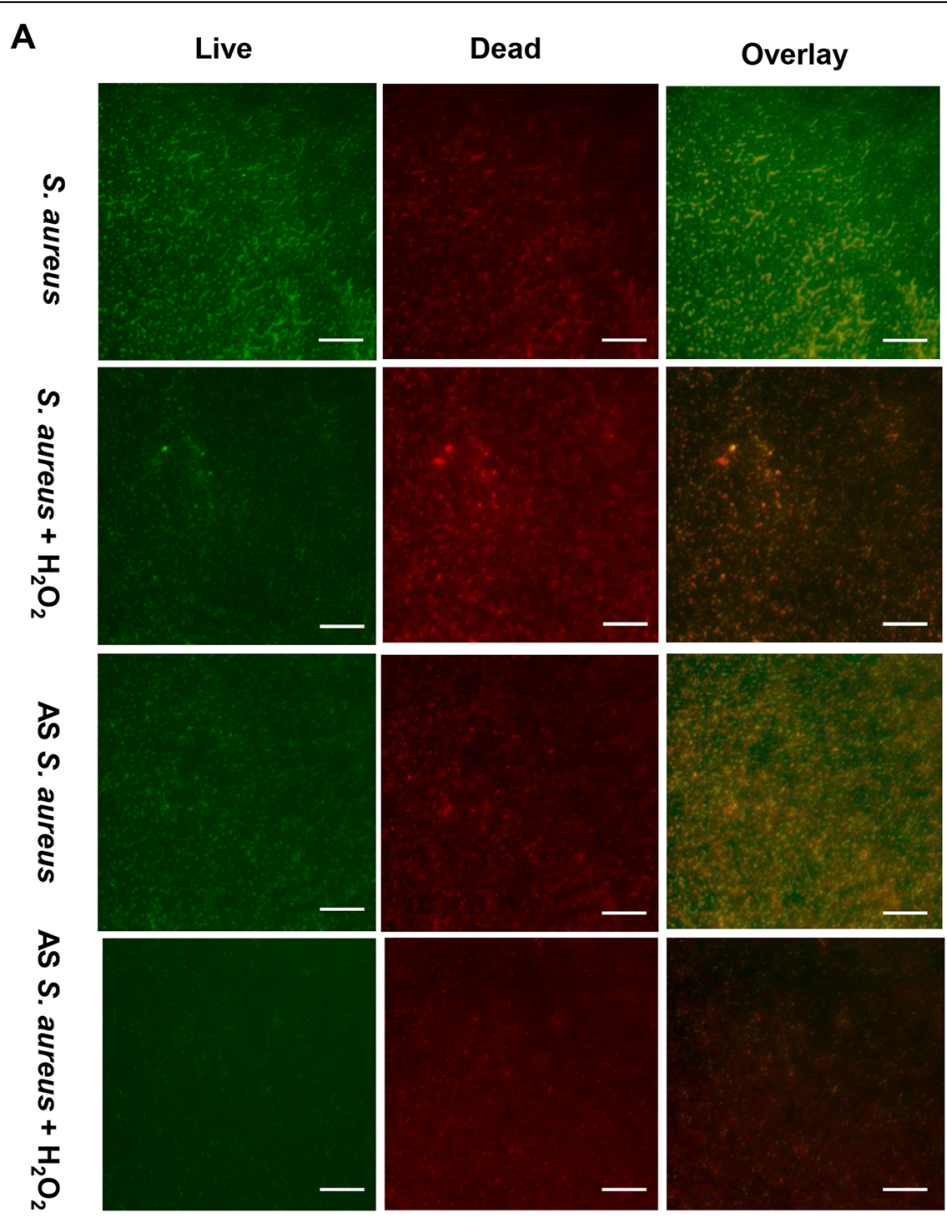

B

\begin{tabular}{ccccc}
\hline & S. aureus & $\begin{array}{c}\text { S. aureus + } \\
\mathrm{H}_{2} \mathrm{O}_{2}\end{array}$ & AS S. aureus & $\begin{array}{c}\text { AS S. aureus } \\
\mathrm{H}_{2} \mathrm{O}_{2}\end{array}$ \\
\hline $\begin{array}{c}\text { Live bacteria } \\
\text { ration (\%) }\end{array}$ & $62.1 \pm 4.2$ & $40.5 \pm 3.6^{*}$ & $46.2 \pm 3.8^{*}$ & $22.1 \pm 4.6^{*}$ \\
\hline
\end{tabular}

Fig. 2 The AS yycG increased susceptibility of the $\mathrm{S}$. aureus to $\mathrm{H}_{2} \mathrm{O}_{2}$. a $\mathrm{S}$. aureus, and $A S y y c G$ strains after $\mathrm{H}_{2} \mathrm{O}_{2}$ treatment. Green, viable bacteria (SYTO 9); red, dead bacteria (PI); scale bars, $100 \mu \mathrm{m}$; b Percentage (\%) of viable S. aureus cells after $\mathrm{H}_{2} \mathrm{O}_{2}$ treatment $\left(n=10\right.$, $\left.{ }^{*} P<0.05\right)$

could directly bind to the promoter regions of the sarA and icaA genes and regulate their expression, which contributed to biofilm metabolism and resistance to antibacterial agents, such as $\mathrm{H}_{2} \mathrm{O}_{2}$ (Fig. 5c).

\section{Discussion}

Microbial biofilm formation is a principal factor for chronic infectious diseases, such as osteomyelitis; therefore, numerous antibiofilm strategies have been devised for clinical use by surgeons, such as aggressive irrigation and physical removal of infective debris [19, 20]. In addition, numerous other promising antibiofilm agents, such as zinc oxide nanoparticles ( $\mathrm{ZnO} \mathrm{NPs}$ ), proteinase $\mathrm{K}$, hamamelitannin (HAM) and antimicrobial peptides (AMPs), have also been developed [21, 22].
Although $3 \% \mathrm{H}_{2} \mathrm{O}_{2}$ is conventionally applied in most bone infection cases to disinfect microorganisms from wounds in clinical practice, the killing ability of $\mathrm{H}_{2} \mathrm{O}_{2}$ on pathogenic bacteria is doubtful [23-25], especially under biofilm conditions in which the resistance increases 1000 fold compared to planktonic bacterial conditions [26-29]. Biofilms, as a physical barrier, could protect microorganisms from the diffusion of environmental stimuli and confer antibiotic resistance to the microorganisms embedded within the biofilm. Multiple factors, including the existence of metabolically inactive bacterial and immune system evasion, contribute to the resistance of biofilms to disinfectant agents [30]. In this study, transcriptome analysis demonstrated that antisense $y y c G$ overexpression downregulated the expression of the oxidative reaction-, 
A

S. aureus

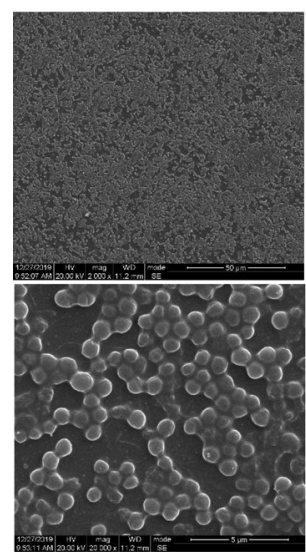

S. aureus $+\mathrm{H}_{2} \mathrm{O}_{2}$
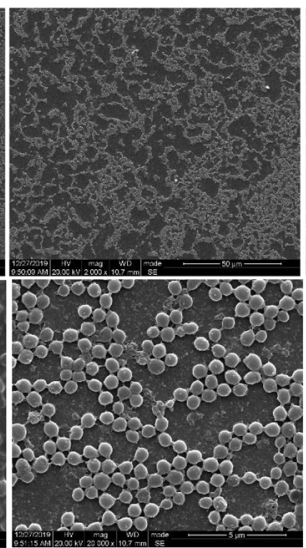

B

\begin{tabular}{lcccc}
\hline & S. aureus & $\begin{array}{c}\text { S. aureus }+ \\
\mathrm{H}_{2} \mathrm{O}_{2}\end{array}$ & AS S. aureus & $\begin{array}{c}\text { AS S. aureus }+ \\
\mathrm{H}_{2} \mathrm{O}_{2}\end{array}$ \\
\hline $\log (\mathrm{CFU}) / \mathrm{mL}$ & $6.2 \pm 0.4$ & $4.34 \pm 0.3^{*}$ & $5.4 \pm 0.3^{*}$ & $3.6 \pm 0.2^{*}$
\end{tabular}

AS S. aureus $+\mathrm{H}_{2} \mathrm{O}_{2}$
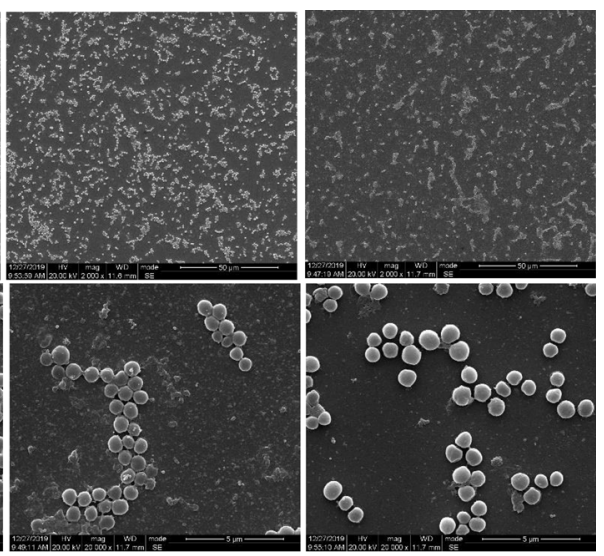

Fig. 3 The AS yycG sensitized biofilm producing S. aureus to $\mathrm{H}_{2} \mathrm{O}_{2}$ intervention synergistically. a SEM images of S. aureus, and ASyycG strains after $\mathrm{H}_{2} \mathrm{O}_{2}$ treatment; b Number of CFUs before and after the $\mathrm{H}_{2} \mathrm{O}_{2}$ treatment $\left[n=10,{ }^{*} P<0.05\right.$, $\left.\lg (\mathrm{CFU} / \mathrm{mL})\right]$

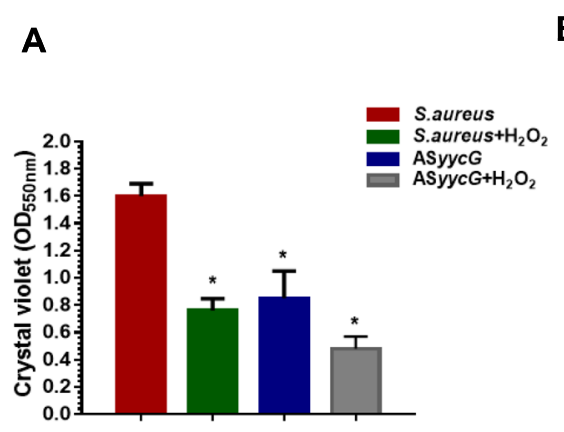

C

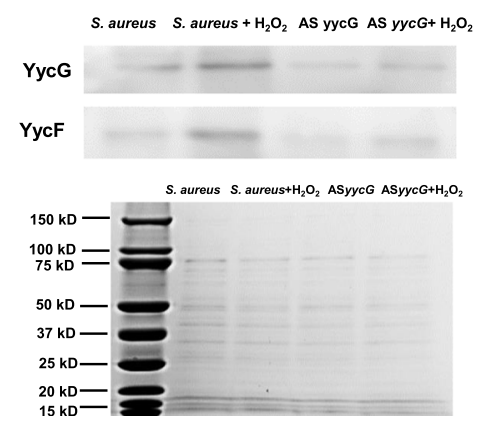

B

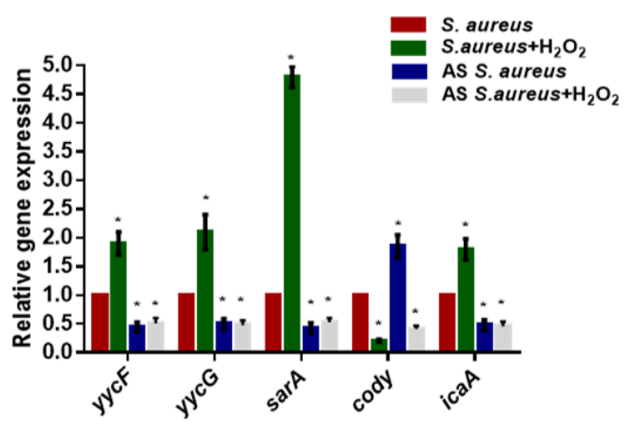

D

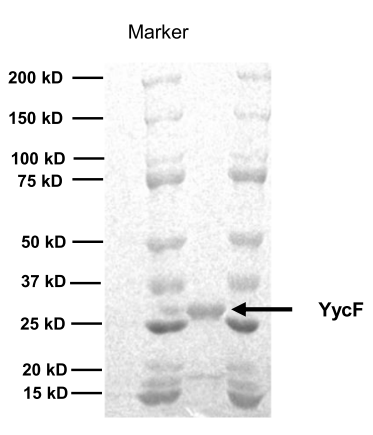

Fig. 4 The AS yycG overexpression inhibited the transcripts of biofilm-related genes. a Biomass was quantified by crystal violet staining. Optical densities at $600 \mathrm{~nm}$ were measured $\left(n=10,{ }^{*} P<0.05\right)$; b Quantitative RT-PCR analysis showed the gene transcripts in $S$. aureus, and ASyycG strains treated with $\mathrm{H}_{2} \mathrm{O}_{2}$. S. aureus gene expression was relatively quantified by RT-PCR using $16 \mathrm{~S}$ as an internal control $\left(n=10,{ }^{*} P<0.05\right)$; c The productions of YycF and YycG were quantified in the cells of S. aureus, and ASyycG strains treated with $\mathrm{H}_{2} \mathrm{O}_{2}$ for Western blotting (upper lane). The lower panel shows a Coomassie-stained gel supporting equal loading of the samples for total bacterial lysis; $\mathbf{d}$ The purified recombinant YycF protein was visualized by Coomassie staining after SDS-PAGE 


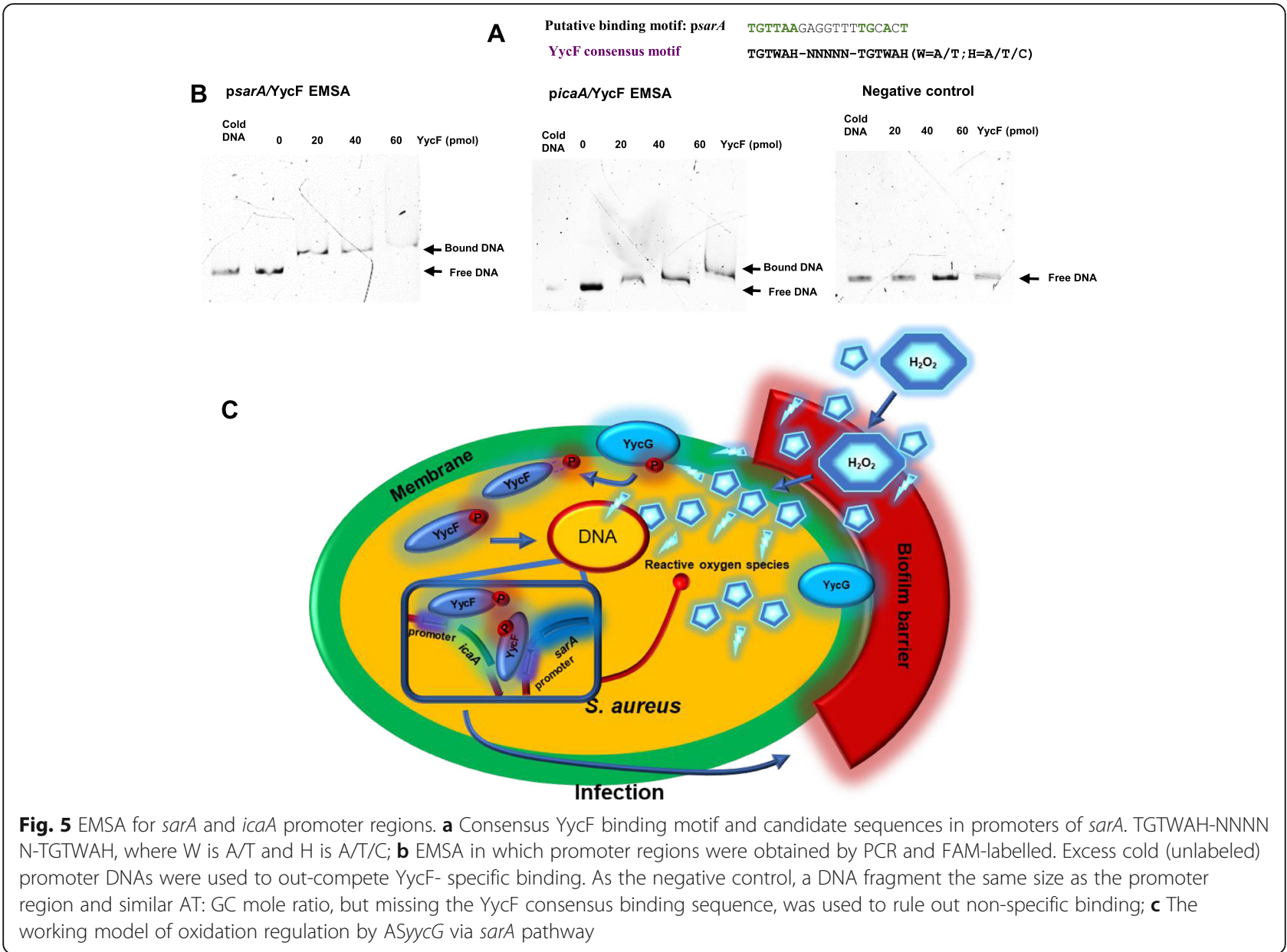

virulence- and biofilm-associated genes ica and sar in $S$. aureus, which may improve the susceptibility of $S$. aureus to $\mathrm{H}_{2} \mathrm{O}_{2}$.

Extracellular polysaccharide substance (EPS), an essential component of biofilms, plays an important role in preventing bacterial colonies from antimicrobial effects. In a previous study, improved killing efficiency of antimicrobial agents for bacteria embedded in EPS could be obtained by combined application with biofilmdispersing enzymes [28, 31]. Our study obtained similar results, which showed that the lowest EPS levels and the fewest cell colony clusters were observed by SEM after $24 \mathrm{~h}$ of culture in the ASyycG $+\mathrm{H}_{2} \mathrm{O}_{2}$ group. The ica $A D B C$ operon synthesized polysaccharide intercellular adhesion (PIA) as a significant component of EPS in biofilm production, which is modulated by the only essential TCS, YycFG, to promote cell wall and biofilm metabolism [32]. A plasmid overexpressing a complementary base-pairing antisense YycG (ASyycG) can significantly reduce biofilm formation by inhibiting the YycFG pathway [33]. Notably, when EPSs are disorganized, the resultant exposure and release of residual biofilm cells to these agents enhance their sensitivity.
Furthermore, the CFU counting assays and live bacterial ratios indicated that $y y c G$ sensitized biofilmproducing $S$. aureus to $\mathrm{H}_{2} \mathrm{O}_{2}$ treatment synergistically. When we combined ASyycG with $\mathrm{H}_{2} \mathrm{O}_{2}$ antibacterial agents, not only was the antibacterial effect of $\mathrm{H}_{2} \mathrm{O}_{2}$ improved, but a prolonged inhibited biofilm formation effect was also acquired $24 \mathrm{~h}$ after treatment with low potential to cause a chronic infection. In vivo research would further be considered to evaluate the synergistic antibacterial effects of ASyycG combined with $\mathrm{H}_{2} \mathrm{O}_{2}$. Although $3 \% \mathrm{H}_{2} \mathrm{O}_{2}$ is continually applied to disinfect wounds microorganisms in clinical irrigation, it oxidizes protein, nucleic acid, and lipids of normal healthy cells, which limited the beneficial effect of promoting wound healing becomes [23, 24]. However, at comparatively low concentrations, the killing ability of $\mathrm{H}_{2} \mathrm{O}_{2}$ on pathogenic bacteria is doubtful $[24,25]$. In the present study, the application of antisense $y y c G$ downregulated the expression of the EPS synthesis-associated gene ica, inhibited biofilm formation and enhanced susceptibility to $\mathrm{H}_{2} \mathrm{O}_{2}$ in $S$. aureus. From this perspective, with the supplementary antisense $y y c G$, an appropriate low therapeutically concentration of $\mathrm{H}_{2} \mathrm{O}_{2}$ could be apply to maintain this 
balance by enhancing antibacterial effect and keeping wound healing with ameliorating wound redox extent.

On the other hand, the sarA gene was reported to enhance biofilm formation by altering ica transcription and the production of PIA in S. aureus [2, 4]. In S. epidermidis, SarA was demonstrated to be an essential positive regulator of the ica operon in biofilm development [8]. In addition, homologous $\mathrm{YycF}$ could directly regulate $i c a A$ and sarA gene expression in S. epidermidis [7]. In the present study, the promoter regions of sarA were directly bound by $\mathrm{YycF}$, as demonstrated via EMSA, which showed that YycF could indirectly regulate ica through the sarA pathway.

Regarding environmental stimuli, the essential TCS YycFG (also known as WalRK) was reported to be associated with bacterial oxidation sensing [34, 35]. Notably, in the present study, the protein production of the cognate sensor kinase $\mathrm{YycG}$, as well as the response regulator YycF, was enhanced in response to $\mathrm{H}_{2} \mathrm{O}_{2}$ intervention, which again confirms that TCS YycFG can be applied to monitor environmental changes. By oxidative stress, biofilm-producing $S$. aureus and the expression of biofilm-inducing genes, such as sarA, were supposed be elevated [35]. However, the expression levels of the antioxidant stress reaction-related and biofilm-associated sarA and ica genes were significantly reduced by ASyycG RNA in the ASyycG strains treated with $\mathrm{H}_{2} \mathrm{O}_{2}$ compared with the parental $S$. aureus ATCC29213 strains in our study. In addition, the global regulator CodY controls the expression of dozens of metabolism and virulence genes in S. aureus, including genes associated with biofilm formation. The activity of CodY in regulating biofilm formation and cell aggregation was associated with $\underline{i c a}$ expression and PIA production [36]. CodY, a regulatory protein, has been reported to repress virulence gene expression in S. aureus [37]. In present study the expression of CodY was relatively high in $A S y y c G$ group, which may contribute to repress the virulence gene expressions in $S$. aureus. However, whether CodY is involved in sensing bacterial oxidation warrants further investigation, particularly concerning the mechanisms by which it regulates virulence genes. Additionally, the regulatory effect of the $\operatorname{sr} A$ gene associated with the YycFG TCS merits further investigation.

\section{Conclusions}

The effects of the antisense $y y c G$ RNA strategy on the susceptibility of biofilm-producing $S$. aureus to $\mathrm{H}_{2} \mathrm{O}_{2}$ treatment were investigated. Recombinant shuttle plasmids were used to overexpress an antisense $y y c G$ RNA $(\mathrm{ASyycG})$ and were transformed into the $S$. aureus strain to construct ASyycG S. aureus. The results of this study indicated that $y y c G$ overexpression inhibited the transcription of biofilm formation-related genes, including
sarA and icaA. Additionally, the CFU counts of ASyycG biofilms treated with $\mathrm{H}_{2} \mathrm{O}_{2}$ were mostly decreased. Notably, YycF could bind to the predicted promoter regions of sarA and icaA genes, demonstrating that susceptibility of biofilm-producing $S$. aureus to $\mathrm{H}_{2} \mathrm{O}_{2}$ intervention may be regulated by ASyycG via the sarA and icaA pathways. In summary, these results indicate that ASyycG synergistically sensitizes biofilm-producing $S$. aureus to $\mathrm{H}_{2} \mathrm{O}_{2}$ treatment and thus may be considered a supplementary strategy for managing osteomyelitis. Considering the possibility of antisense $y y c G$ breakdown by $\mathrm{H}_{2} \mathrm{O}_{2}$, a stabilizing vector, such as nanographene oxide, may be utilized in future research to support the clinical strategy of combining $\mathrm{H}_{2} \mathrm{O}_{2}$ with antisense $y y c G$.

\section{Methods}

\section{Bacterial strains and biofilms growth conditions}

S. aureus strain ATCC29213, provided by the Department of Laboratory Medicine (West China Hospital, Sichuan University, Chengdu, China), was cultured in tryptic soy broth (TSB) [16]. In a previous study, we performed phenotypic experiments to ensure that $S$. aureus strain ATCC29213 was a biofilm-producing pathogen [33]. After overnight incubation at $37{ }^{\circ} \mathrm{C}$ and $5 \% \mathrm{CO}_{2}$, $500 \mu \mathrm{L}$ of $S$. aureus suspension was inoculated into 10 $\mathrm{mL}$ fresh TSB medium to mid-logarithmic phase (optical density at $\left.600 \mathrm{~nm}\left[\mathrm{OD}_{600}\right]=0.5\right)$. For the formation of biofilms, sterilized glass disks (diameter of $10 \mathrm{~mm}$ ) were dropped into log-phased S. aureus sterile 24-well microtiter plates for 24-h coculture biofilms. This basic culture section was prepared for the subsequent tests.

\section{Construction of S. aureus antisense yycG overexpression strains}

To observe the effect of antisense $y y c G$ in $S$. aureus, we first constructed antisense $y y c G$-overexpressing $S$. aureus strains according to the following protocols. Recombination of the plasmid pDL278 containing the antisense $y y c G$ fragment was conducted by inserting the antisense $y y c G$ (ASyycG) sequence into restriction sites between BamHI and EcoRI, which was subsequently synthesized by Sangon Biotech (Shanghai, China). According to our previous protocol [38], an ASyycG-overexpressing $S$. aureus (ASyycG mutants) was constructed by adding 200 ng recombinant pDL278 ASyycG plasmid with $1 \mu \mathrm{g} /$ $\mathrm{mL}$ competence-stimulating peptide (CSP) into a $250-\mu \mathrm{L}$ mid-exponential-phase $S$. aureus suspension for $60 \mathrm{~min}$ [33]. The empty pDL278 plasmid did not exert any effects on the viability of $S$. aureus [39].

\section{RNA extraction analyses}

To investigate the alterations in the whole transcriptome between S. aureus ATCC29213 and ASyycG mutants, whole transcriptome analysis was applied. First, samples 
of S. aureus strain ATCC29213 and ASyycG mutants were cultured in $10 \mathrm{~mL}$ of fresh TSB media into the mid-logarithmic phase. Total RNA was isolated and quantified as previously described [33]. Agarose gel electrophoresis $(1 \%)$ was run to check for degradation or contamination of the RNA. We used a NanoPhotometer ${ }^{\circ}$ spectrophotometer (IMPLEN, CA, USA) for RNA quality and quantity checks. The integrity of the extracted RNA sample was assessed using the RNA Nano 6000 Assay Kit of the Bioanalyzer 2100 system (Agilent Technologies, CA, USA).

\section{Library preparation for whole transcriptome sequencing} After the abovementioned RNA extraction, exactly $5 \mu \mathrm{g}$ RNA per sample for the S. aureus ATCC29213 and ASyycG mutant groups was used as input for library preparation. Sequencing libraries were generated using the NEBNext ${ }^{\circ}$ Ultra $^{\text {Th }}$ RNA Library Prep Kit for Illumina ${ }^{\circ}$ (NEB, USA) following the manufacturer's recommendations. Next, index codes were added to attribute sequences to each sample. The rRNA was removed using a specialized kit. Fragmentation was performed using divalent cations under elevated temperature in NEBNext First Strand Synthesis Reaction Buffer (5X). Using a fragmented mRNA template, first-strand cDNA was synthesized using random hexamer primers in the M-MuLV Reverse transcription system. The RNA was digested by $\mathrm{RNase} \mathrm{H}$, and then second strand cDNA synthesis was subsequently performed using DNA Polymerase I and RNase $\mathrm{H}$. The remaining overhangs were converted into blunt ends via exonuclease/polymerase activities. After adenylation of the 3' ends of DNA fragments, NEBNext adapter with a hairpin loop structure was ligated. Next, USER Enzyme (NEB, USA) was employed to degrade the second strand of cDNA. The library fragments were purified using the AMPure XP system (Beckman Coulter, Beverly, USA) to select cDNA fragments with a length of $250 \sim 300 \mathrm{bp}$. Then, PCR was performed using Phusion High-Fidelity DNA polymerase, universal PCR primers, and Index (X) Primer. Similarly, the above PCR products were purified (AMPure XP system), and library quality was assessed on the Agilent Bioanalyzer 2100 system. The cluster generation of the index-coded samples was performed on a cBot Cluster Generation System using TruSeq PE Cluster Kit v3-cBot-HS (Illumina) according to the manufacturer's instructions. After clustering, sequencing was performed on an Illumina NovaSeq platform, whereby 150 -bp paired-end reads were generated.

\section{Differential expression analysis}

Following the above library preparation, differential expression between the two groups was analyzed. In this analysis, high-quality clean data were obtained by eliminating reads with adapters and low-quality reads from raw data. Bowtie 2 was applied to map the abovefiltered reads to the reference genome. To quantify the gene expression level, HTSeq v0.6.1 was used to count the read numbers mapped to each gene. Next, the number of fragments per kilobase of transcript sequence per million base pairs sequenced (FPKM) of each gene was calculated. Differential expression analysis of two groups was performed using the DESeq R package (1.18.0) [40]. DESeq provided statistical routines for determining differential expression in digital gene expression data using the negative binomial distribution-based model. The resulting $P$-values were adjusted using Benjamini and Hochberg's approach to control the false discovery rate. Genes with adjusted $P$-values $<0.05$ found via DESeq were classified as differentially expressed.

\section{KEGG enrichment analysis of differentially expressed genes}

After the procedure described above was performed, raw data regarding differentially expressed genes were acquired. To identify the functions of differentially expressed genes between the S. aureus and ASyycG groups, Kyoto Encyclopedia of Genes and Genomes (KEGG) enrichment analysis was applied. KEGG is a database resource for elucidating high-level functions and utilities of biological systems, such as cells, organisms, and ecosystems, from molecular-level information, especially large-scale molecular datasets generated by genome sequencing and other high-throughput experimental technologies (http://www.genome.jp/kegg/) [41]. In this study, we used KOBAS software to test the statistical enrichment of differentially expressed genes in KEGG pathways. The results showed 20 KEGG pathways with adjusted P-values of approximately 0.5, involving the $S$. aureus infection pathway, and enrichment score differences were detected between the antisense $y y c G$ overexpression group and ATCC29213 group.

\section{Bacterial exposure to oxidants}

After genetic alternation analysis and associated functional enrichment screening, we examined biofilm characteristics associated with resistance strength to chemical situlas, particularly $\mathrm{H}_{2} \mathrm{O}_{2}$, by overexpressing ASyycG. To determine the susceptibility of $S$. aureus ATCC29213 and ASyycG mutants to oxidants, 24-h-old biofilms derived from these groups were treated with $3 \% \mathrm{H}_{2} \mathrm{O}_{2}$ (for clinical irrigation, $\mathrm{H}_{2} \mathrm{O}_{2}$ is usually $3 \%$ ) for 30 min at $37{ }^{\circ} \mathrm{C}$. Residual $\mathrm{H}_{2} \mathrm{O}_{2}$ was removed by diluting biofilm samples in PBS three times [23, 42]. The biofilm sample disks were cultured in TSB medium for $24 \mathrm{~h}$ after treatment. 


\section{Microtiter dish assay and colony-forming unit (CFU) counting}

The microtiter dish assay was applied to examine the biomass of 24-h-old biofilms after interventions with crystal violet $(\mathrm{CV})$. For the formation of biofilms, samples of S. aureus strain ATCC29213 and ASyycG mutants were cultured into the midlogarithmic phase in 1 $\mathrm{mL}$ TSB media, which were dropped into sterile 24-well microtiter plates for $24 \mathrm{~h}$. After $24 \mathrm{~h}$ of culture, the culture medium was removed. We washed the established biofilms three times with PBS solution. The dye bound to the biofilms was assayed after $0.1 \%(\mathrm{w} / \mathrm{v})$ crystal violet staining for $15 \mathrm{~min}$ followed by $1 \mathrm{~mL}$ destaining solution (ethanol/acetone $=8: 2$ ). Subsequently, $100 \mu \mathrm{L}$ of the solution was transferred into a new 96-well plate, and the absorbance was measured with a microplate reader (ELX800, Gene) at $\mathrm{OD}_{600} \mathrm{~nm}$ [33].

For the CFU counting test, the treated biofilms were subsequently cultured in TSB medium. After $24 \mathrm{~h}$ of culturing, the biofilms were collected via ultrasound in 1 $\mathrm{mL}$ of PBS for $15 \mathrm{~min}$. The acquired microbiological samples were serially diluted 6-fold (from $10^{-1}$ to $10^{-6}$ ) in PBS and spread onto TSA agar plates for incubation at $37{ }^{\circ} \mathrm{C}$ in $5 \% \mathrm{CO}_{2}$ for $24 \mathrm{~h}$. To determine cell viability, $\mathrm{CFU}$ per millimeter of microbiological suspension was counted [16].

Furthermore, the biofilms treated for $24 \mathrm{~h}$ were labeled with SYTO9 (LIVE/DEAD Bacterial Viability Kit reagent; BacLight, Invitrogen, Grand Island, NY, USA); live cells appeared green, while dead cells were stained red with propidium iodide. The cells were visualized using epifluorescence microscopy (Nikon Eclipse TE-2000 S, Melville, NY) at $40 \times$ magnification. Notably, five random fields in each specimen were selected [16].

\section{Characterizing biofilm morphology}

Scanning electron microscopy (SEM) was applied to assess the structure of biofilms. The samples were diluted twice using PBS and fixed with $2.5 \%$ glutaraldehyde for 4 h. Fixed samples were serially dehydrated with increasing concentrated ethanol solutions from 30 to $100 \%$ and dried using a critical point dryer. Subsequently, samples were coated with gold powder. We obtained micrographs using a scanning electron microscope (Inspect, Hillsboro, OR, USA) [16].

\section{Analysis of gene expression using quantitative real-time PCR}

To further validate the biofilm-associated genes and YycFG pathway modulated by ASyycG according to the enrichment results, quantitative real-time PCR was conducted according to the following protocols. Total RNA was extracted and purified with the MasterPure ${ }^{\mathrm{Tm}}$ RNA Purification Kit (Epicentre Technologies, Epicentre,
Madison, WI, USA) from 24-h-treated biofilm samples obtained from the $S$. aureus group, $S$. aureus $+\mathrm{H}_{2} \mathrm{O}_{2}$ group, AS yycG group, and AS yycG $+\mathrm{H}_{2} \mathrm{O}_{2}$ group following the manufacturer's instructions. Contaminating genomic DNA was digested and removed with Turbo RNase-free DNase I (Ambion, New York, NY USA) according to the recommended instructions. Next, the purity (A260/A280) and concentration of RNA were determined using a NanoDrop 2000 spectrophotometer (Thermo Scientific, Waltham, MA, USA). The purified RNA was reverse-transcribed to cDNA using random hexamers or gene-specific primers (Table 1) with the RevertAid First Strand cDNA Synthesis Kit (Thermo Scientific). We conducted quantitative real-time polymerase chain reaction (qRT-PCR) assays using the primers listed in Table 1, with the 16Sr RNA gene serving as an internal control $[16,33]$. Each sample was analyzed in triplicate, and the threshold cycle values (CT) were quantified.

\section{Western blotting}

To determine the protein production of $\mathrm{YycF}$ and $\mathrm{YycG}$, Western blotting was performed as described below. $S$. aureus ATCC 29,213 and ASyycG mutants were cultured to the mid-logarithmic phase (optical density at $600 \mathrm{~nm}[\mathrm{OD} 600]=0.5)$. Then, we treated the $S$. aureus planktonic cultures with $100 \mathrm{mM} \mathrm{H}_{2} \mathrm{O}_{2}$ for $60 \mathrm{~min}$, washed them and resuspended them in PBS ( $\mathrm{pH}$ 7.2). The bacterial cells, including the $S$. aureus group, $S$. aureus $+\mathrm{H}_{2} \mathrm{O}_{2}$ group, AS yycG group, and AS yycG $+\mathrm{H}_{2} \mathrm{O}_{2}$ group, were mechanically disrupted as previously described [31]. For Western blot analysis, equal amounts of protein $(30 \mu \mathrm{g})$ were mixed with $2 \mathrm{X}$ SDS-PAGE Sample Loading Buffer (Sangon Biotech, Shanghai, China) in boiling water for $10 \mathrm{~min}$ and loaded on $10 \%$ SDS-PAGE gels (Bio-Rad). Proteins were fractionated and electrotransferred to polyvinylidene fluoride (PVDF) membranes (Biosharp Biotech, Shanghai, China). Membranes were blocked in TSBT buffer $(100 \mathrm{mM}$ Tris- $\mathrm{HCl}, 2.5$ $\mathrm{mM} \mathrm{NaCl}$ ) containing $5 \% \mathrm{w} / \mathrm{v}$ nonfat dry milk at room temperature for $2 \mathrm{~h}$. To measure YycG and YycF production, membranes were incubated with purified YycGand YycF-specific antibodies (1:500, HuaBio Biotechnology, Hangzhou, China) for $2 \mathrm{~h}$ at room temperature, washed in Tris-buffered saline containing $0.1 \%$ Tween 20 , and incubated with horseradish peroxidase (HRP)conjugated goat anti-rabbit secondary antibody $(1: 5,000)$ for $2 \mathrm{~h}$ at room temperature as previously described [28]. Protein immunoreactive bands were visualized using an Immobilon Western Chemiluminescent kit (Millipore, Billerica, MA, USA). We utilized a Coomassie-stained gel to estimate equal loading of the samples for total bacterial lysis. 
Table 1 Sequences of primers in this study

\begin{tabular}{|c|c|c|}
\hline Primers & sequence 5'-3' (Forward/Reverse) & Reference \\
\hline \multicolumn{3}{|l|}{ RT-qPCR } \\
\hline \multirow[t]{2}{*}{$i c a A$} & 5'- GATTATGTAATGTGCTTGGA -3'/ & Ref. [30] \\
\hline & 5'- ACTACTGCTGCGTTAATAAT - 3' & \\
\hline \multirow[t]{2}{*}{ yycF } & $5^{\prime}$ - TGGCGAAAGAAGACATCA -3'/ & Ref. [30] \\
\hline & $5^{\prime}$ - AACCCGTTACAAATCCTG- $3^{\prime}$ & \\
\hline \multirow[t]{2}{*}{ yycG } & 5' - CGGGGCGTTCAAAAGACTTT -3'/ & Ref. [30] \\
\hline & $5^{\prime}$ - TCTGAACCTTTGAACACACGT - $3^{\prime}$ & \\
\hline \multirow[t]{2}{*}{ sarA } & 5' - AGATGGCCCTTCTTCAAATG -3'/ & This study \\
\hline & $5^{\prime}$-CCGCAATAATTCTTGTGACG -3' & \\
\hline \multirow[t]{2}{*}{$\operatorname{cod} Y$} & $5^{\prime}$ - GGTGGAGGGGAAAGATTAGG -3'/ & This study \\
\hline & 5' -GCGCGCTTCTITTTCTACTT -3' & \\
\hline \multirow[t]{2}{*}{165 rRNA } & $5^{\prime}$ - GTAGGTGGCAAGCGTTATCC -3'/ & Ref. [30] \\
\hline & $5^{\prime}$-CGCACATCAGCGTCAACA-3' & \\
\hline \multicolumn{3}{|l|}{ EMSA assay } \\
\hline \multirow[t]{2}{*}{ promoter regions of sarA } & 5' - GCGCAATTTGGTGAAGTTTGATAGATG -3'/ & This study \\
\hline & $5^{\prime}$-GTGATATATAAACCTAGGGCATAAAGTCC - $3^{\prime}$ & \\
\hline \multirow[t]{2}{*}{ promoter regions of icaA } & $5^{\prime}$ - CTGAAAATTAATCACACTATGTTACAGG -3'/ & This study \\
\hline & $5^{\prime}$ - CTITACCTACCTTCGTTAGTTAGGTTG -3' & \\
\hline
\end{tabular}

The program Primer3 (https://bioinfo.ut.ee/primer3-0.4.0/) was used to design the $\operatorname{sar} A$ and $\operatorname{cod} Y$ primers. For the sarA gene, the forward primer targeted starting at nucleotide 194, and the reverse primer targeted starting at nucleotide 429 . For the codY gene, the forward primer targeted starting at nucleotide 352 , and the reverse primer targeted starting at nucleotide 501

\section{Electrophoretic mobility shift assay (EMSA)}

To determine whether the YycF protein could directly bind to the promoter regions of the sarA and icaA genes, electrophoretic mobility shift assays (EMSAs) were conducted. To generate YycFHis-Tag fusion proteins, the ORF was amplified and restricted. Then, purified products were cloned into digested pET-22b (Novagen) to yield pET-yycG by Huabio Biotech (Hangzhou, China). Plasmids were transformed into $E$. coli BL21. Recombinant proteins were isolated from 500 $\mu \mathrm{L}$ of culture after a 3 -h induction with $1 \mathrm{mM}$ IPTG. After cell lysis, recombinant proteins were purified through affinity chromatography on $\mathrm{Ni}^{2+}$ NTA agarose (Qiagen) as previously described [43]. The purified protein was visualized via Coomassie staining after SDSPAGE.

EMSA was used to determine whether the sarA or $i c a A$ gene was directly regulated by $\mathrm{YycF}$ as previously described [43] with modifications. A PCR amplicon was generated from $S$. aureus ATCC29213 genomic DNA using primers labeled with the 5' FAM (Roche) (see Table 1). Before the binding reaction, DNA fragments were purified according to the manufacturer's instructions (Tiangen Biotech, Beijing, China). Labeled DNA fragments $(0.02 \mathrm{pmol})$ were incubated with increasing amounts of recombinant $\mathrm{YycF}(0,20,40$, and $60 \mathrm{pmol})$ and a 100-fold excess of unlabeled DNA fragments (cold
DNA) as a competitor. The reaction mixture $(20 \mu \mathrm{L})$ contained $50 \%$ glycerinum, labeled DNA fragments, and YycF proteins.

After 20 min incubation at room temperature, samples were loaded on native PAGE gels in $0.5 \times$ TBE buffer (44.5 mM Tris- $\mathrm{HCl}, 44.5 \mathrm{mM}$ boric acid, $1 \mathrm{mM}$ EDTA, pH 8.0). Native PAGE was prepared using $5 \times$ TBE $(445$ $\mathrm{mM}$ Tris- $\mathrm{HCl}, 445 \mathrm{mM}$ boric acid, $10 \mathrm{mM}$ EDTA, $\mathrm{pH}$ 8.0), $30 \%$ Acr-Bis (29:1), $50 \%$ glycerinum, $10 \%$ ammonium persulfate (APS), and N,N,N',N'-tetramethylethylenediamine (TEMED). Gel electrophoresis was performed at $80 \mathrm{~V}$ and $4{ }^{\circ} \mathrm{C}$ for $90 \mathrm{~min}$ on ice.

\section{Data analysis}

All statistical data were analyzed in SPSS 16.0 (SPSS Inc., Chicago, IL, USA). The Shapiro-Wilk test was used to analyze the distribution of data, and the Bartlett test was used to determine the homogeneity of variances. For parametric testing, we adopted one-way ANOVA to assess the statistical significance of variables followed by the Tukey test. In the data, $P$-values $<0.05$ were considered to indicate significant differences.

\section{Abbreviations}

S. aureus: Staphylococcus aureus; ASyycG:antisense yycG RNA; PIA: Polysaccharide intercellular adhesion; S. epidermidis:Staphylococcus epidermidis": TCSs: two-component signal transduction systems; RR:response regulator;" ASRNA: Antisense RNA; EMSA:electrophoretic mobility shift assay;" 
EPS: Extracellular polysaccharide substance; PIA:Polysaccharide intercellular adhesion; TSB:Tryptic soy broth; CSP:Competence stimulating peptide:" KEGG: Kyoto Encyclopedia of Genes and Genomes; CV:Crystal violet;" CFU: Colony-forming units; SEM:Scanning electron microscopy;" qRT-

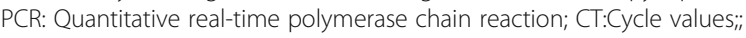
PVDF: Polyvinylidene fluoride; APS:Ammonium persulfate;" TEME D: tetramethylethylenediamine; sarA: Staphylococcal accessory regulator;" yycG: membrane-bound sensor histidine kinase associated with cell wall metabolism.

\section{Supplementary Information}

The online version contains supplementary material available at https://doi. org/10.1186/s12866-021-02218-x.

Additional file 1:Supplementary Figure 1. The productions of $Y y c G$ (A) and YycF (B)were quantified in the groups of S. aureus, S. aureus + $\mathrm{H} 2 \mathrm{O} 2, \mathrm{AS}$ yycG, and $\mathrm{AS}$ yycG + $\mathrm{H} 2 \mathrm{O} 2$ for Western blotting.

Supplementary Figure 2. Coomassie-stained gel supporting equal loading of the samples for total bacterial lysis (A). The purified recombinant YycF protein was visualized by Coomassie staining after SDS-PAGE (B).

\section{Acknowledgements}

Not applicable.

\section{Authors' contributions}

SZW, YJL, HZ, and LL were responsible for the study design and the interpretation of results. SZW and LL were responsible for manuscript preparation. All authors read and approved the final manuscript. $L L$ and $H Z$ contributed equally to this paper (co-corresponding authors).

\section{Funding}

This work was supported by the Sichuan Provincial Natural Science Foundation of China (Grant No. 2021YJ0455 and 2018SZ0125), Post-Doctor Research Project, West China Hospital, Sichuan University (Grant No. $2020 \mathrm{HXBH} 134)$. The funders had no role in the design of the study and collection, analysis, and interpretation of data and in writing the manuscript. The authors have no other financial relationships relevant to this article to disclose.

\section{Availability of data and materials}

The data that support the findings of this study are available from the corresponding author upon reasonable request.

\section{Declarations}

Ethics approval and consent to participate

Not applicable.

\section{Consent for publication}

Not applicable.

\section{Competing interests}

The authors have no conflicts of interest to disclose in relation to this article.

\section{Author details}

${ }^{1}$ Department of Orthopedics, West China Hospital, Sichuan University, No.37 Guoxue Alley, Sichuan 610041 Chengdu, P.R. China. ${ }^{2}$ West China School of Public Health, Sichuan University, Chengdu, China. ${ }^{3}$ Department of Preventive Dentistry, Hospital of Stomatology, State Key Laboratory of Oral Diseases, Sichuan University, NO.14 Third Section, Renmin South Road, Sichuan 610041 Chengdu, P.R. China.

Received: 21 October 2020 Accepted: 5 May 2021

Published online: 30 May 2021

\section{References}

1. Beavers WN, Skaar EP. Neutrophil-generated oxidative stress and protein damage in Staphylococcus aureus. Pathog Dis. 2016;74(6):ftw060.
2. Liu L, Shen X, Yu J, Cao X, Zhan Q, Guo Y, et al. Subinhibitory Concentrations of Fusidic Acid May Reduce the Virulence of S. aureus by Down-Regulating sarA and saeRS to Reduce Biofilm Formation and a-Toxin Expression. Front Microbiol. 2020;11:25.

3. Sun F, Liang H, Kong X, Xie S, Cho H, Deng X, et al. Quorum-sensing agr mediates bacterial oxidation response via an intramolecular disulfide redox switch in the response regulator AgrA. Proc Natl Acad Sci U S A. 2012; 109(23):9095-100.

4. Valle J, Toledo-Arana A, Berasain C, Ghigo JM, Amorena B, Penadés JR, et al. SarA and not sigmaB is essential for biofilm development by Staphylococcus aureus. Mol Microbiol. 2003;48(4):1075-87.

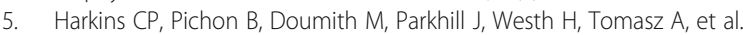
Methicillin-resistant Staphylococcus aureus emerged long before the introduction of methicillin into clinical practice. Genome Biol. 2017;18(1):130.

6. Rupp ME, Fey PD, Heilmann C, Götz F. Characterization of the importance of Staphylococcus epidermidis autolysin and polysaccharide intercellular adhesin in the pathogenesis of intravascular catheter-associated infection in a rat model. J Infect Dis. 2001;183(7):1038-42.

7. Xu T, Wu Y, Lin Z, Bertram R, Götz F, Zhang Y, et al. Identification of Genes Controlled by the Essential YycFG Two-Component System Reveals a Role for Biofilm Modulation in Staphylococcus epidermidis. Front Microbiol. 2017:8:724

8. Tormo MA, Martí M, Valle J, Manna AC, Cheung AL, Lasa I, et al. SarA is an essential positive regulator of Staphylococcus epidermidis biofilm development. J Bacteriol. 2005;187(7):2348-56.

9. Cheung AL, Projan SJ. Cloning and sequencing of sarA of Staphylococcus aureus, a gene required for the expression of agr. J Bacteriol. 1994;176(13): 4168-72.

10. Bayer MG, Heinrichs JH, Cheung AL. The molecular architecture of the sar locus in Staphylococcus aureus. J Bacteriol. 1996;178(15):4563-70.

11. Cheung AL, Eberhardt $K$, Heinrichs JH. Regulation of protein A synthesis by the sar and agr loci of Staphylococcus aureus. Infect Immun. 1997;65(6): 2243-9.

12. Hall JW, Yang J, Guo H, Ji Y. The Staphylococcus aureus AirSR TwoComponent System Mediates Reactive Oxygen Species Resistance via Transcriptional Regulation of Staphyloxanthin Production. Infect Immun. 2017:85(2):e00838-16.

13. Hall JW, Ji Y. Sensing and Adapting to Anaerobic Conditions by Staphylococcus aureus. Adv Appl Microbiol. 2013:84:1-25.

14. Villanueva M, García B, Valle J, Rapún B, Ruiz de Los Mozos I, Solano C, et al. Sensory deprivation in Staphylococcus aureus. Nat Commun. 2018:9(1):523.

15. Dubrac S, Msadek T. Identification of genes controlled by the essential YycG/YycF two-component system of Staphylococcus aureus. J Bacteriol. 2004;186(4):1175-81.

16. Wu S, Huang F, Zhang H, Lei L. Staphylococcus aureus biofilm organization modulated by YycFG two-component regulatory pathway. J Orthop Surg Res. 2019;14(1):10.

17. Wu S, Lin K, Liu Y, Zhang H, Lei L. Two-component signaling pathways modulate drug resistance of Staphylococcus aureus (Review). Biomed Rep. 2020;13(2):5

18. Wu S, Liu Y, Zhang H, Lei L. Nano-graphene oxide improved the antibacterial property of antisense yycG RNA on Staphylococcus aureus. J Orthop Surg Res. 2019;14(1):305.

19. Høiby N, Bjarnsholt T, Moser C, Bassi GL, Coenye T, Donelli G, et al. ESCMID guideline for the diagnosis and treatment of biofilm infections 2014. Clin Microbiol Infect. 2015;21:1-25.

20. Koo H, Allan RN, Howlin RP, Stoodley P, Hall-Stoodley L. Targeting microbial biofilms: current and prospective therapeutic strategies. Nat Rev Microbiol. 2017;15(12):740-55.

21. Abd El-Hamid MI, El-Naenaeey Y, Kandeel ES,M, Hegazy T, Mosbah WAH, Nassar RA. MS, et al. Promising Antibiofilm Agents: Recent Breakthrough against Biofilm Producing Methicillin-Resistant Staphylococcus aureus. Antibiotics (Basel). 2020;9(10):667.

22. Batoni G, Maisetta G, Esin S. Antimicrobial peptides and their interaction with biofilms of medically relevant bacteria. Biochim Biophys Acta. 2016 1858(5):1044-60

23. Zhu G, Wang Q, Lu S, Niu Y. Hydrogen Peroxide: A Potential Wound Therapeutic Target? Med Princ Pract. 2017;26(4):301-8.

24. Yamada Y, Mokudai T, Nakamura K, Hayashi E, Kawana Y, Kanno T, et al. Topical treatment of oral cavity and wounded skin with a new disinfection system utilizing photolysis of hydrogen peroxide in rats. J Toxicol Sci. 2012; 37(2):329-35 
25. Thomas GW, Rael LT, Bar-Or R, Shimonkevitz R, Mains CW, Slone DS, et al. Mechanisms of delayed wound healing by commonly used antiseptics. J Trauma. 2009;66(1):82-90.

26. Parsek MR, Singh PK. Bacterial biofilms: an emerging link to disease pathogenesis. Annu Rev Microbiol. 2003;57:677-701.

27. Rasmussen TB, Givskov M. Quorum sensing inhibitors: a bargain of effects. Microbiology. 2006;152(Pt 4):895-904.

28. Roy R, Tiwari M, Donelli G, Tiwari V. Strategies for combating bacterial biofilms: A focus on anti-biofilm agents and their mechanisms of action. Virulence. 2018;9(1):522-4.

29. Wu H, Moser C, Wang HZ, Høiby N, Song ZJ. Strategies for combating bacterial biofilm infections. Int J Oral Sci. 2015;7(1):1-7.

30. Hathroubi S, Mekni MA, Domenico P, Nguyen D, Jacques M. Biofilms: Microbial Shelters Against Antibiotics. Microb Drug Resist. 2017;23(2):147-56.

31. Darouiche RO, Mansouri MD, Gawande PV, Madhyastha S. Antimicrobial and antibiofilm efficacy of triclosan and DispersinB combination. J Antimicrob Chemother. 2009;64(1):88-93.

32. Dubrac S, Boneca IG, Poupel O, Msadek T. New insights into the WalKWalR (YycG/YycF) essential signal transduction pathway reveal a major role in controlling cell wall metabolism and biofilm formation in Staphylococcus aureus. J Bacteriol. 2007;189(22):8257-69.

33. Wu S, Liu Y, Lei L, Zhang H. Antisense yycG Regulation of Antibiotic Sensitivity of Methicillin-Resistant Staphylococcus aureus in Chronic Osteomyelitis. Surg Infect (Larchmt). 2019;20(6):472-9.

34. Senadheera MD, Lee AW, Hung DC, Spatafora GA, Goodman SD, Cvitkovitch DG. The Streptococcus mutans vic X gene product modulates gtfB/C expression, biofilm formation, genetic competence, and oxidative stress tolerance. J Bacteriol. 2007;189(4):1451-8.

35. Kulkarni R, Antala S, Wang A, Amaral FE, Rampersaud R, Larussa SJ, et al. Cigarette smoke increases Staphylococcus aureus biofilm formation via oxidative stress. Infect Immun. 2012;80(11):3804-11.

36. Mlynek KD, Bulock LL, Stone CJ, Curran LJ, Sadykov MR, Bayles KW, et al. Genetic and Biochemical Analysis of CodY-Mediated Cell Aggregation in Staphylococcus aureus Reveals an Interaction between Extracellular DNA and Polysaccharide in the Extracellular Matrix. J Bacteriol. 2020;202(8): e00593-19.

37. Queiroux C, Bonnet M, Saraoui T, Delpech P, Veisseire P, Rifa E, et al. Dialogue between Staphylococcus aureus SA15 and Lactococcus garvieae strains experiencing oxidative stress. BMC Microbiol. 2018;18(1):193.

38. Wu S, Liu Y, Zhang H, Lei L. A new transformation method with nanographene oxides of antisense carrying yycG RNA improved antibacterial properties on methicillin-resistant Staphylococcus aureus biofilm. J Vet Med Sci. 2019;81(10):1540-6.

39. Wu S, Liu Y, Zhang H, Lei L. The Susceptibility to Calcium Hydroxide Modulated by the Essential walR Gene Reveals the Role for Enterococcus faecalis Biofilm Aggregation. J Endod. 2019;45(3):295-301.

40. Wang L, Feng Z, Wang X, Wang X, Zhang X. DEGseq: an R package for identifying differentially expressed genes from RNA-seq data. Bioinformatics. 2010;26(1):136-8

41. Kanehisa M, Araki M, Goto S, Hattori M, Hirakawa M, Itoh M, et al. KEGG for linking genomes to life and the environment. Nucleic Acids Res. 2008;36: 480-4.

42. Pang YY, Schwartz J, Bloomberg S, Boyd JM, Horswill AR, Nauseef WM Methionine sulfoxide reductases protect against oxidative stress in Staphylococcus aureus encountering exogenous oxidants and human neutrophils. J Innate Immun. 2014;6(3):353-64.

43. Lei L, Stipp RN, Chen T, Wu SZ, Hu T, Duncan MJ. Activity of Streptococcus mutans VicR Is Modulated by Antisense RNA. J Dent Res. 2018;97(13):147784

\section{Publisher's Note}

Springer Nature remains neutral with regard to jurisdictional claims in published maps and institutional affiliations.

Ready to submit your research? Choose BMC and benefit from:

- fast, convenient online submission

- thorough peer review by experienced researchers in your field

- rapid publication on acceptance

- support for research data, including large and complex data types

- gold Open Access which fosters wider collaboration and increased citations

- maximum visibility for your research: over $100 \mathrm{M}$ website views per year

At BMC, research is always in progress.

Learn more biomedcentral.com/submissions 